\title{
TRADUÇÃO E ADAPTAÇÃO DA ESCALA BIS/BAS PARA APLICAÇÃO EM ADULTOS BRASILEIROS
}

Ellen PORTILHO-SOUZA, Clínica Contexto e Pontifícia Universidade Católica de Goiás, eportilho88@yahoo.com.br. Claudio Herbert NINA-E-SILVA, Laboratório de Psicologia Anomalística e Neurociências, Universidade de Rio Verde.

\section{Recebido em: 30/10/2013 - Aprovado em: 20/12/2013 - Disponibilizado em: 15/01/2014}

Resumo: O temperamento pode ser compreendido como um padrão de comportamento que diferencia as pessoas entre si e as habilita a enfrentar as situações do cotidiano de forma característica e própria. Em algumas teorias o temperamento tem sido caracterizado como fundamento biológico no qual a personalidade se estrutura. No que se refere à avaliação do temperamento, pode-se afirmar que existem poucos instrumentos traduzidos e validados no Brasil. Os instrumentos disponíveis, em sua maioria, são somente traduzidos, mas não adaptados à cultura brasileira, como é o caso da Pavlovian Temperament Survey. O presente trabalho objetivou realizar a tradução e adaptação transcultural da escala norte-americana BIS/BAS de avaliação do temperamento (CARVER \& WHITE, 1994). Os estágios da pesquisa foram: tradução, retrotradução, avaliação/equivalência literal, idiomática e semântica entre a tradução e a retrotradução, elaboração da versão síntese, aplicação da versão síntese, cálculo dos coeficientes de correlação dos itens em inglês e português da escala e cálculo do Alfa de Cronbach. Aproximadamente 79\% dos itens da escala apresentaram índices fortes de correlação positiva, não havendo nenhum itens com correlação não-significativa. O Alfa de Cronbach calculado foi de 0,686 .

Palavras-chave: temperamento, personalidade, escala psicológica, psicobiologia.

Abstract: Temperament can be understood as a pattern of behavior that differentiates people and enables them to cope with everyday situations individually and properly. In some theories temperament has been characterized as a biological foundation in which the personality is based. As regards the assessment of temperament, it can be stated that there are few instruments translated and validated in Brazil. The available tools, in most cases, are only translated, but not adapted to the Brazilian culture, as is the case of the Pavlovian Temperament Survey. This study aimed to perform the translation and cultural adaptation of the American BIS / BAS scale of assessment of temperament (CARVER \& WHITE, 1994). The stages of the research were : translation , back translation, evaluation literal equivalence, idiomatic and semantics between the translation and back-translation, preparation of the synthetic version , the application of the synthetic version, calculation of correlation coefficients of the items in English and Portuguese and calculation of the scale Cronbach's Alpha coefficient. Approximately $79 \%$ of the scale items had strong positive correlation coefficient, with no items with no significant correlation . The Cronbach's Alpha was calculated at 0.686 .

Keywords: temperament, personality, psychological scale, psychobiology.

\section{Introdução}

O conceito de temperamento tem sido considerado útil na análise funcional dos comportamentos-problema por vários psicoterapeutas (FARMER, 2005). A principal razão para isso residiria no acúmulo de evidências empíricas de que o temperamento estaria relacionado às diferenças individuais na sensibilidade ao reforço e à punição (GRAY \& BRAVER, 2002; FARMER, 2005; BOKSEM et al, 2006).

Segundo Rothbart e Bates (1998),
alguns dos construtos teóricos mais
empregados no estudo do temperamento são

os modelos que enfatizam a base biológica do temperamento. Dentre esses modelos, um dos mais aplicados em pesquisa clínica é a teoria psicobiológica da personalidade de Gray (1970; 1987). De acordo com esse modelo, há duas dimensões de personalidade, a ansiedade e a impulsividade. Segundo Gray (1987), as características de personalidade representam as diferenças individuais na sensibilidade de dois sistemas neurológicos nas repostas a estímulos ambientais relevantes. O sistema subjacente à ansiedade é o sistema de inibição comportamental (BIS - behavioral inhibition system), e o sistema subjacente à impulsividade é um sistema de ativação 
comportamental (BAS - behavioral activation system).

De acordo com Gray (1987), o BIS é sensível aos sinais de punição, novidade, e não recompensa. Este sistema, então, inibe comportamentos que podem levar a resultados negativos ou dolorosos. O funcionamento do BIS é responsável pela experiência de sentimentos negativos como medo, ansiedade, frustração e tristeza, em resposta aos estímulos. O BAS, por sua vez, é sensível aos sinais de recompensa e fuga de punição. $\mathrm{O}$ funcionamento do BAS é responsável pela experiência de sentimentos positivos como elação, felicidade e esperança (CARVER \& WHITE, 1994).

De acordo com Hall, Lindzey e Campbell (2000), pode-se dizer que a discussão de Gray diz respeito, principalmente, à suscetibilidade às pistas de recompensa e punição como característica comportamental subjacente nos indivíduos impulsivos e ansiosos.

A BIS/BAS Scale (CARVER \& WHITE, 1994) é um instrumento de avaliação do temperamento, composta por 24 itens, que foi desenvolvida com o objetivo de refletir as diferenças individuais quanto à sensibilidade aos sistemas regulatórios neurofisiológicos propostos por Gray. O BIS, sistema de inibição comportamental, e é subjacente à ansiedade. O BAS, sistema de ativação comportamental, por sua vez, está subjacente à impulsividade.
O participante deve analisar os itens da BIS/BAS Scale considerando o que mais se assemelha com seu pensamento e comportamento e classificar cada item de acordo com uma escala do tipo Likert de quatro pontos: $1=$ muito verdadeiro para mim; $2=$ mais verdadeiro do que falso para mim; $3=$ mais falso do que verdadeiro para mim; 4=totalmente falso para mim. Os itens são divididos de acordo com os sistemas BIS e BAS, anteriormente descritos, e os itens considerados filtros. Os itens pertencentes ao domínio de BIS são: $2^{*}, 8,13,16,19,22 *$ e 24. Os itens considerados filtros são: 1, 6, 11 e 17. Quanto ao sistema BAS, a escala permite o cálculo de escores parciais referentes a três subdomínios deste sistema, sendo eles: BAS Drive (itens 3, 9, 12 e 21), $B A S$ Fun Seeking (itens 5, 10, 15 e 20), BAS Reward Reponsiveness (itens 4, 7, 14, 18 e 23).

No que se refere à pertinência da tradução e adaptação da BIS/BAS Scale para o português, em primeira mão podemos citar a escassez de instrumentos para avaliação do temperamento. Além disso, os instrumentos disponíveis, se comparados a BIS/BAS Scale, possuem um número maior de itens o que inviabiliza sua aplicação em alguns casos.

$\mathrm{Na}$ adaptação cultural de instrumentos busca-se a equivalência semântica, idiomática, experiencial e conceitual da versão traduzida e adaptada com a versão original (MATTOS, SERRA-PINHEIROS, ROHDE \& PINTO, 2006). Para haver 
equivalência semântica as palavras devem ter os mesmos significados e não existirem múltiplos significados para um mesmo item. Para que haja equivalência idiomática, é necessário o emprego de estratégias para tradução de itens que possuem coloquialismo e expressões idiomáticas e não podem ser traduzidos literalmente. Itens com experiências vivenciadas no cotidiano de um cultura podem ser modificados para obter uma equivalência experiencial, ou seja, devem ser substituídos por experiências específicas da cultura para a qual o instrumento está sendo adaptado. Quanto à equivalência conceitual, consiste na verificação de conceitos que podem ser utilizados de formas diferentes entre culturas.

A importância de se buscar equivalência entre a versão em língua estrangeira e o português tem sido cada vez mais reconhecida, crescendo o número de estudos que, em diferentes campos, buscam a criação de instrumentos considerando essa preocupação. (MATTOS, SERRAPINHEIROS, ROHDE \& PINTO, 2006). Desse modo, o presente trabalho objetivou realizar a tradução e adaptação transcultural da escala norte-americana BIS/BAS de avaliação do temperamento (CARVER \& WHITE, 1994).

\section{Materiais e Métodos}

O trabalho foi dividido em duas etapas, sendo a primeira dividida em sete estágios e a segunda etapa dividida em três estágios. Os procedimentos das etapas serão descritos separadamente.

\section{Etapa 1}

Nesta etapa do estudo os participantes foram divididos em duas amostras. A primeira amostra foi composta por 7 participantes, com conhecimento da língua inglesa, pertencentes à rede social dos pesquisadores. A segunda amostra foi composta por 12 participantes, estudantes de Psicologia. A participação foi consentida voluntariamente e, no caso da segunda amostra, mediante a assinatura do Termo de Consentimento Livre e Esclarecido (TCLE). O projeto foi aprovado pelo Comitê de Ética em Pesquisa da instituição a que pertenciam os pesquisadores.

A primeira etapa consistiu em sete estágios descritos a seguir. No primeiro estágio, a versão original da BIS/BAS Scale foi traduzida por dois pesquisadores conhecedores da língua inglesa, separadamente. No segundo estágio, foi feita a comparação entre as traduções diretas realizadas na primeira etapa. Neste estágio, além dos tradutores, uma terceira pesquisadora participou das comparações e análise qualitativa dos itens traduzidos. O resultado do segundo estágio foi uma versão traduzida preliminar que, no segundo estágio, foi encaminhada para um tradutor de origem norte-americana, fluente em português e em inglês, que realizou sua retrotradução.

O quarto estágio consistiu na avaliação da equivalência literal, idiomática e semântica 
entre a tradução e a retrotradução. A partir das análises realizadas neste estágio foi obtida uma nova versão da escala denominada versão síntese.

O quinto estágio consistiu na aplicação das versões original e síntese em uma amostra bilíngüe composta por adultos pertencentes à rede social dos pesquisadores (Amostra 1). A partir dos dados obtidos neste estágio, foram calculados os coeficientes de correlação dos itens em inglês e português da escala.

A versão síntese foi aplicada em uma amostra de 12 participantes (Amostra 2), sendo esse o sexto estágio da primeira etapa. O sétimo estágio consistiu no cálculo do Alfa de Cronbach tanto dos dados obtidos na amostra bilíngüe, quanto dos dados obtidos na sexta etapa.

\section{Etapa 2}

Nesta segunda etapa do estudo, participaram uma especialista na língua inglesa, 10 participantes bilingues (Amostra 3) e 35 alunos de Psicologia (Amostra 4). A participação foi consentida voluntariamente e, no caso da Amostra 4, mediante a assinatura do Termo de Consentimento Livre e Esclarecido (TCLE).

No primeiro estágio da segunda etapa as versões original e síntese foram apresentadas a uma especialista em língua inglesa no intuito de melhoramento na equivalência semântica, idiomática e conceitual de alguns itens. A partir das considerações feitas pela especialista e o consenso entre os pesquisadores os itens que apresentaram correlações não significativas na primeira etapa do estudo (itens 1, 4, 8, 10, 16, 18, 20 e 22) foram modificados e foi feita a versão final.

O segundo estágio da segunda etapa consistiu na aplicação das versões original e final em uma amostra de 10 participantes bilíngües (Amostra 3) e cálculo do coeficiente de correlação a partir dos dados obtidos.

O terceiro estágio consistiu no cálculo do alfa de Cronbach a partir dos dados coletados com a aplicação da escala na Amostra 4.

\section{Resultados e Discussão}

\subsection{Análises qualitativas da tradução e retrotradução dos itens.}

Os dados referentes ao estágios 1 a 4 da Etapa 1 consistiram em dados qualitativos obtidos a partir da comparação das versões tradução direta e reversa da escala BIS BAS. Dos 24 itens que compõem a escala, identificou-se a necessidade de aperfeiçoamento da tradução do item 10. A tradução do item 10 ("I will often do things for no other reason than that they might be fun") obtida no segundo estágio do procedimento na retrotradução resultou em " $I$ usually only do things that give me pleasure", e então considerada inapropriada pelos pesquisadores e especialista bilíngüe que realizaram a retrotradução. Foi feita a modificação de "Eu geralmente só faço coisas que me dêem prazer" para "Eu geralmente 
faço coisas por nenhuma outra razão a não ser pela diversão". Após esta modificação foi elaborada a versão síntese, como mostra o Anexo 1.

\subsection{Análise da consistência das respostas} da amostra 1 aos itens das versões originais e em português da escala.

Com base nos dados obtidos no estágio 5 da Etapa 1, foram obtidos 24 coeficientes de correlações a partir da associação entre os itens originais e os itens traduzidos. Cinco itens evidenciaram índices fortes de correlação $(0,80<$ rho $\leq 1)$, onze itens apresentaram correlações moderadas e significativas $(0,40<$ rho $\leq 0,80)$ e oito itens apresentaram correlações não significativas, como mostra o Anexo 1.

\subsection{Avaliação da confiabilidade da escala.}

O sexto e sétimo estágio da Etapa 1 tiveram como objetivo avaliar a confiabilidade da escala pelo cálculo do alfa de Cronbach. A partir dos dados da Amostra 1, o alfa de Cronbach mostrou valores acima de 0,8 tanto na versão em inglês quanto na versão em português $(0,829$ e $\quad 0,859$ respectivamente). Já nos dados obtidos a partir da aplicação da versão síntese na Amostra 2, o alfa de Cronbrach foi 0,482.

A partir dos resultados obtidos na Etapa 1do procedimento foi constatada a necessidade de realização dos estágios da Etapa 2, descritos a seguir.

\subsection{Resultados da Etapa 2.}

$\mathrm{O}$ item 1 "A person's family is the most important thing in life", que foi traduzido, na versão síntese, como " 1 . A família de uma pessoa é a coisa mais importante na vida", na versão final optou-se por "1. A família é a coisa mais importante na vida". Essa mudança ocorreu devido a possibilidade de interpretação da frase como se dirigida a família de terceiros, o que não seria o objetivo de avaliação da escala.

$\mathrm{O}$ item 4 "When I'm doing well something I love to keep at it", que, na versão síntese, foi traduzido como "4. Quando estou indo bem em alguma coisa eu adoro permanecer nisso até o fim", na versão final optou-se por “4. Quando as coisas estão dando certo para mim, eu continuo nelas tranquilamente". Percebeu-se que, na versão original em inglês, a sentença procura avaliar a permanência em alguma atividade reforçadora. Na versão síntese, a sentença poderia sinalizar persistência para alguns indivíduos, por isso optou-se pela mudança acima descrita.

O item "8. Criticism or scolding hurts me quite a bit", na versão síntese traduzido como "8. Críticas ou repreensão me magoam demais.", foi modificado na versão final para "8. Críticas me magoam bastante". A substituição da palavra "demais" por "bastante" ocorreu para que houvesse a possível diminuição de uma conotação de "extremamente ruim" que a palavra "demais" pode ocasionar. A palavra "repreensão" foi 
retirada pois, culturalmente, ela pode representar possível punição

$\mathrm{O}$ item "10. I will often do things for no other reason than that they might be fun", traduzido na versão síntese como "10. Eu geralmente faço coisas por nenhuma outra razão a não ser pela diversão”, foi modificado para "10. Muitas vezes, eu procuro fazer coisas que pareçam ser divertidas".

$\mathrm{O}$ item "16. If I think something unpleasant is going to happen I usually get pretty worked up”, na versão síntese traduzido como "16.Quando eu acho que alguma coisa ruim está para acontecer eu geralmente fico preocupado", foi modificado para "16. Quando eu acho que alguma coisa desagradável está para acontecer eu geralmente fico preocupado" na versão final.

$\mathrm{O}$ item "18. When good things happen to me, it affects me strongly" traduzido inicialmente, na versão síntese, da seguinte maneira: "18. Eu fico muito emocionado quando coisas boas acontecem comigo e isso me afeta muito", foi modificado para " 18 . Quando coisas boas acontecem, isso mexe muito comigo". Optou-se pela mudança pois a palavra "afeta" não é utilizada na cultura brasileira como na norte-americana, não havendo diferença quanto ao objetivo de avaliação da sentença após a mudança.

$\mathrm{O}$ item "20. I crave excitement and new sensations", na versão síntese traduzido "20. Eu preciso de novas emoções e novas sensações", foi modificado para "20.Eu desejo novas emoções e novas sensações". A sentença se refere, segundo os autores da escala, ao comportamento apetitivo, sendo incluída no subdomínio Fun Seeking do sistema BAS. Os pesquisadores concordaram que o desejo/querer/procurar novas emoções se incluíram neste subdomínio do sistema BAS.

Por fim, o item "22. I have very few fears compared to my friends", na versão síntese como "22. Eu tenho menos medos do que os meus amigos", na versão final optou-se por "22. Comparando com os meus amigos, eu tenho poucos medos". $\mathrm{Na}$ opinião dos pesquisadores, a tradução da versão final (Anexo 2) aproximou-se mais do sentido da versão original da sentença em inglês.

Os resultados do terceiro estágio da segunda etapa do estudo mostraram um aumento no alfa de Cronbach em relação a primeira etapa do estudo, de 0,482 para 0,686.

\section{Conclusão}

Pode-se concluir que o presente trabalho atingiu os objetivos propostos e apresenta uma adaptação brasileira da Escala BIS/BAS para avaliação do temperamento. Vale ressaltar a importância de processo acurado e minucioso de tradução, adaptação cultural e validação para manutenção de acuidade em relação à escala original. Recomenda-se a realização de estudos sobre validade de critério e de construto da escala adaptada bem como a aplicação em diferentes estados para avaliação de possíveis diferenças 
no desempenho da escala o que possibilitará o uso da escala de modo mais confiável tanto em pesquisa quanto no contexto clínico.

\section{Referências Bibliográficas}

BOKSEM, M.A.S.; TOPS, M.; WESTERA, A.E.; MEIJMANA, T.F.; LORIST, M.M. Error-related ERP components and individual differences in punishment and reward sensitivity. Brain Research, 1001, p.92-101.

CARVER, C. S.; WHITE, T. L. Behavioral inhibition, behavioral activation, and affective responses to impeding reward and punishment: The BIS/BAS Scale. Journal of Personality and Social Psychology, 67, p. 319-333, 1994.

GRAY, J. A. The psychophysiological basis of introversion-extraversion. Behavior Research and Therapy, 8, p.249-266, 1970.

GRAY, J. A. Perspectives on anxiety and impulsivity: A commentary. Journal of Research in Personality, 21(4), p.493-509, 1987.

GRAY, J. R.; BRAVER, T.S. Personality predicts working-memory-related activation in the caudal anterior cingulate cortex. Cognitive, Affective and Behavioral Neuroscience, 2(1), p.64-75, 2002.

HALL, C. S.; LINDZEY, G.; CAMPBELL, J. B. (2000). Teorias da Personalidade. 4 ed., Porto Alegre: Artmed, 2000. .

MATTOS, P.; SERRA-PINHEIRO, M.A.; ROHDE, L.A.; PINTO, D. Apresentação de uma versão em português para uso no Brasil do instrumento MTA-SNAP-IV de avaliação de sintomas de transtorno do déficit de atenção/hiperatividade e sintomas de transtorno desafiador e de oposição. Revista de Psiquiatria do Rio Grande do Sul. v. 28, n. 3, p. 290-297, 2006.
ROTHBART, M. K.; BATES, J. E. (1998). Temperament. In DAMON, W. (Series Ed.) N. EISENBERG (Vol. Ed.), Handbook of child psychology: Vol. 3. Social, emotional and personality development. New York: Wiley, p. 105-176, 1998. 